\title{
More on Simpson's paradox and the analysis of memory retrieval
}

\author{
LARS NYBERG \\ University of Umeå, Umeå, Sweden \\ (Lars-Göran Nilsson, Sponsor)
}

\begin{abstract}
A common way of analyzing the statistical relation between two tests of memory is to use contingency analyses. A potential problem with such analyses is known as Simpson's paradox. The paradox is that collapsing two or more contingency tables may have the effect that the relationship expressed in the overall contingency table differs from the relationships expressed in the original tables. The paradox arises when covariates are correlated with each of the tests. It has been claimed that the paradox has implications for the analysis of memory retrieval, and ways of solving the problem have been called for (Hintzman, 1980). In this paper, partial gamma (Davis, 1967 ) is suggested as one possible solution. This method can be used to compute a weighted average of the results in the original tables. The use of partial gamma is exemplified by applying it to hypothetical instances of Simpson's paradox.
\end{abstract}

Analyzing the statistical relation between the performance on two successive tests of memory has for a long time been an important method for studying memory processes (e.g., Martin, 1971; Tulving \& Watkins, 1975). More recently, this method has also been applied to the study of the relation between explicit and implicit forms of memory (e.g., Hayman \& Tulving, 1989).

A common way of estimating the statistical relation between two successive tests of memory is to use $2 \times 2$ contingency analyses. Yule's $Q$ has been suggested as an appropriate measure of association in such $2 \times 2$ tables (Hayman \& Tulving, 1989). Yule's $Q$ is a special version of gamma (Goodman \& Kruskal, 1954) that is applicable on $2 \times 2$ tables only. The range of $Q$ is -1 to 1 , with 0 indicating lack of association. For values in between 0 and 1 , a significance test of $Q$ exists (Hayman \& Tulving, 1989).

A potential problem with $2 \times 2$ contingency analyses is known as Simpson's paradox. The paradox means that if two or more contingency tables are collapsed into one, the relationship expressed in the summary table may differ from the original tables (Simpson, 1951). Simpson's paradox can take many different forms (Hintzman, 1980). For instance, if two events, $X$ and $Y$, are correlated in the same direction with a covariate, $Z$, and $X$ and $Y$ are positively related at levels $Z 1$ and $Z 2$, the summary table will show a stronger relationship between $X$ and $Y$ than is existing at $Z 1$ and $Z 2$. If, on the other hand, $X$ and $Y$ are negatively correlated at levels $Z 1$ and $Z 2$, this negative rela-

Thanks are due Fergus I. M. Craik, Arthur J. Flexser, John M. Gardiner, Douglas L. Hintzman, Lars-Göran Nilsson, Ulrich Olofsson, and Endel Tulving for comments on earlier versions of this article. Correspondence concerning this article should be addressed to L. Nyberg, Department of Psychology, University of Umeå, S-901 87 Umeå, Sweden. tionship can be reduced, nullified, or even reversed in the summary table. One form of the paradox is illustrated numerically in Table 1 (cf. Hintzman, 1980, Table 1).

In Table 1 , contingency tables at two levels of $Z$ are given for variables $X$ and $Y$ (the original tables) together with a summary table across $Z$. The relationship between $Z$ and $X$ is negative, and the relationship between $Z$ and $Y$ is positive. $Q$ values are presented for both the original tables and the summary table.

Table 1 illustrates Simpson's paradox by showing a reversal of two positive contingencies to a negative contingency in the summary table. This reversal is caused by the opposite correlations between $Z$ and $X$, and $Z$ and $Y$. It is thus apparent that the summary table, in this case, does not adequately summarize the relationships expressed in the original tables. Conclusions of the relationship between $X$ and $Y$ based on the summary table, ignoring the influence of $Z$, may consequently be misleading.

It is important to realize that the outcomes in both the original tables and in the summary table are correct; they are merely two aspects of the same thing (cf. Martin, 1981). The result that should be considered depends on the question asked. For example, in one case it might be of interest to find out the relationship between $X$ and $Y$ for only some levels of $Z$, and in another case it might be the overall relation that is of interest. In still other cases, it might be proper to have a coefficient that adequately summarizes the results in the original tables (i.e., that gives an estimate of the contingency relation between $X$ and $Y$ when $Z$ is controlled). The purpose of the present paper is to introduce such a coefficient.

The particular coefficient considered here is a partial coefficient that has been developed for gamma (Davis, 1967). Partial gamma summarizes the results in various test strata, which in this case apply to various levels of 
Table 1

Illustration of One Form of Simpson's Paradox

\begin{tabular}{|c|c|c|c|c|}
\hline \multirow[b]{2}{*}{$Z$} & \multirow[b]{2}{*}{$X$} & \multicolumn{2}{|c|}{$Y$} & \multirow[b]{2}{*}{$Q$} \\
\hline & & - & + & \\
\hline $1(+)$ & $\begin{array}{l}+ \\
-\end{array}$ & $\begin{array}{r}2 \\
20\end{array}$ & $\begin{array}{l}18 \\
60\end{array}$ & .50 \\
\hline $2(-)$ & $\begin{array}{l}+ \\
-\end{array}$ & $\begin{array}{l}90 \\
48\end{array}$ & $\begin{array}{l}50 \\
12\end{array}$ & .44 \\
\hline Total & $\begin{array}{l}+ \\
-\end{array}$ & $\begin{array}{l}92 \\
68\end{array}$ & $\begin{array}{l}68 \\
72\end{array}$ & -.18 \\
\hline
\end{tabular}

$Z$ (Davis, 1967, considers the case where both the events $[X, Y]$ and the variable to be held constant $[Z]$ are categorized). It is thus possible to calculate the statistical relation between two variables and, at the same time, control for the influence of a third variable.

The formula for ordinary gamma is defined as $(a d-b c) /$ $(a d+b c)$, where $a, b, c$, and $d$ denote the,,+++--+ , and - - cells of a contingency table. To calculate partial gamma, the contingency table is stratified according to the levels of the covariate $(Z)$ into $n$ component tables, where the $i$ th table has entries $a_{i}, b_{i}, c_{i}$, and $d_{i}$. The $a$ cell in the summary table is the summation over $i$ of all the $a_{i} \mathrm{~s}$, and similarly for the other three cells. The formula for the partial gamma coefficient is then defined as

$$
G_{X, Y \mid Z}=\frac{\sum\left(a_{i} d_{i}-b_{i} c_{i}\right)}{\sum\left(a_{i} d_{i}+b_{i} c_{i}\right)},
$$

where the summations are over $i$. The partial coefficient is, thus, obtained by summing the numerators and denominators of all the component gammas, and dividing one sum by the other. Ordinary gamma and partial gamma are consequently the same when $n=1$.

The calculation of partial gamma will be illustrated by applying this coefficient on the data presented in Table 1 . First, the numerators and denominators of all the component gammas are to be calculated and summed. This calculation is shown in Table 2 .

The partial gamma coefficient for the relationship between $X$ and $Y$ is then obtained by dividing the sums:

$$
G_{X, Y \mid Z}=\frac{1560}{3960}=.39 .
$$

The value of the partial gamma coefficient is in line with the values expressed in the original tables, showing a positive contingency between $X$ and $Y$. Note that a greater weight is given to the stratum with the larger number of frequencies; partial gamma can also be considered as a weighted average of the conditional correlations in the var- ious test strata. The weight is "the proportion of the total pairs differing on $A$ and $B$ and tied on $C$ which are in that stratum" (Davis, 1967, p. 191). For further discussion of the weights used, see Quade (1974).

The example above shows that, in contrast to the $Q$ value based on the summary table, partial gamma adequately summarizes the results in the original tables. This coefficient should therefore be appropriate to use when a covariate to $X$ and $Y$ is identified and one intends to calculate the contingency relation between $X$ and $Y$ with the covariate controlled (note that it is possible to control for the effect of multiple covariates and any interaction effect of particular covariate combinations on the $X, Y$ relationship; cf. Davis, 1967).

A critical issue is the identification of relevant covariates. Hintzman (1980) illustrated the problem of Simpson's paradox by taking examples from the memory literature. In the examples, it was assumed that the investigator only had the summary table to work from and that the nature of the component tables are unknown. Hintzman then assumed the presence of different types of covariates (i.e., subject differences, item differences, and subject-item interactions) and presented hypothetical examples of how Simpson's paradox can complicate the interpretation of the results presented in the summary tables. Consider one of the examples given by Hintzman (1980); independent retrieval in the A-B, A-C paradigm. In this paradigm, subjects study two paired-associate lists that have an A-B, $A-C$ relationship and are later tested for recall of $B$ and $C$ using $A$ as retrieval cue. Several studies have shown independence between $B$ and $C$ recall (e.g., Martin, 1971). This independence contradicts the negative relationship predicted by the associative interference theory. Hintzman (1972) argued that the failure of a negative relation to show up may be caused by factors producing positive correlations between $B$ and $C$ recall. Subject differences were given as a hypothetical example of such a factor; good subjects are likely to recall both B and C, whereas the opposite is true for poor subjects. In Table 3, data for this hypothetical example is presented along with values of gamma.

Note that $B$ and $C$ are negatively related at each level of the covariate, but the value of gamma based on the summary table is one of independence; the negative relationship is masked by the positive correlations between B and $C$ recall. The partial gamma coefficient for the relationship between B and C when ability is controlled is then

$$
G_{B, C \text { lability }}=\frac{-17500}{37500}=-.47 \text {. }
$$

Table 2

Illustration of Calculation of Partial Gamma for Data in Table 1

\begin{tabular}{ccccc}
\hline$Z$ & $a \times d$ & $b \times c$ & $a d-b c$ & $a d+b c$ \\
\hline 1 & $18 \times 20=360$ & $60 \times 2=120$ & $360-120=240$ & $360+120=480$ \\
2 & $50 \times 48=2400$ & $12 \times 90=1080$ & $2400-1080=1320$ & $2400+1080=3480$ \\
& & $\Sigma(a d-b c)=1560$ & $\sum(a d+b c)=3960$ \\
\hline
\end{tabular}


Table 3

Recall Performance of Hypothetical Subjects of High, Medium, and Low Ability (Adopted From Hintzman, 1972, Table 1)

\begin{tabular}{|c|c|c|c|c|}
\hline \multirow[b]{2}{*}{ Ability } & \multirow[b]{2}{*}{ B } & \multicolumn{2}{|c|}{$\mathrm{C}$} & \multirow[b]{2}{*}{$Q$} \\
\hline & & - & + & \\
\hline \multirow[t]{2}{*}{ Low (+) } & + & 50 & 0 & -1.0 \\
\hline & - & 150 & 50 & \\
\hline \multirow[t]{2}{*}{ Medium } & + & 150 & 100 & -.38 \\
\hline & - & 100 & 150 & \\
\hline \multirow[t]{2}{*}{ High (-) } & + & 50 & 150 & -1.0 \\
\hline & - & 0 & 50 & \\
\hline \multirow[t]{2}{*}{ Total } & + & 250 & 250 & 0 \\
\hline & - & 250 & 250 & \\
\hline
\end{tabular}

Thus, when ability is controlled, the negative relation between $B$ and $C$ predicted by the associative interference theory is found. However, given the assumption by Hintzman (1980) that the nature of the component tables are unknown to the investigator (i.e., that the covariate is "'hidden"), this effect of subject ability on the relationship between $B$ and $C$ recall would have remained unnoticed and the negative relation between $B$ and $C$ would not have been found.

The identification of critical covariates requires evaluation at the level of single experiments, and a few attempts, in which an overall contingency table has been broken down into a number of component tables to study the effect of potential covariates, have been reported in the literature (see, e.g., Martin, 1981). In such cases, par- tial gamma should be useful to summarize the results in the various component tables, and comparisons of the partial coefficient with the zero order coefficient will reveal how the relationship between two variables is affected when the covariate is controlled.

\section{REFERENCES}

Davis, J. A. (1967). A partial coefficient for Goodman and Kruskal's Gamma. American Statistical Association Journal, 62, 189-193.

Goodman, L. A., \& KrusKal, W. H. (1954). Measures of association for cross classifications. Journal of the American Statistical Association, 54, 732-764.

Hayman, C. A., \& Tulving, E. (1989). Contingent dissociation between recognition and fragment completion: The method of triangulation. Journal of Experimental Psychology: Learning, Memory, \& Cognition, 15, 228-240.

Hintzman, D. L. (1972). On testing the independence of associations. Psychological Review, 79, 261-264.

HintzMan, D. L. (1980). Simpson's paradox and the analysis of memory retrieval. Psychological Review, 87, 398-410.

MARTIN, E. (1971). Verbal learning theory and independent retrieval phenomena. Psychological Review, 78, 314-332.

MARTIN, E. (1981). Simpson's paradox resolved: A reply to Hintzman. Psychological Review, 88, 372-374.

QuADE, D. (1974). Nonparametric partial correlation. In H. M. Blalock, Jr. (Ed.), Measurements in the social sciences: Theories and strategies (pp. 369-398). Chicago: Aldine.

Simpson, E. H. (1951). The interpretation of interaction in contingency tables. Journal of the Royal Statistical Society, Series B, 13, 238-241.

Tulving, E., \& WATkins, M. J. (1975). Structure of memory traces. Psychological Review, 82, 261-275.

(Manuscript received April 14, 1993.) 